Artículo Original

\title{
Medidas adicionales de higiene y bioseguridad a consecuencia de la pandemia COVID-19 en la industria peruana
}

\section{Additional hygiene and biosafety measures as a result of the COVID-19 pandemic in Peruvian industry}

https://doi.org/10.52808/bmsa.7e5.61e2.009

Roberto Carlos Dávila Morán ${ }^{1, *}$ https://orcid.org/0000-0003-3181-8801

Leonardo Velarde Dávila ${ }^{2}$

https://orcid.org/0000-0002-8096-0196

Héctor Portillo Rios ${ }^{3}$

https://orcid.org/0000-0003-1432-8472

José Leonor Ruiz Nizama ${ }^{4}$

https://orcid.org/0000-0003-0444-244X

Fernando Vásquez Perdomo ${ }^{4}$

https://orcid.org/0000-0002-0537-447X

Recibido: 12/02/2021

Aceptado: 23/06/2021

\section{RESUMEN}

El alto riesgo de contagio y afectación por COVID-19 en los trabajadores de la industria hizo necesario replantear los protocolos de higiene y bioseguridad ya establecidos, con medidas como el distanciamiento en puestos de trabajo, el escalonamiento en horario de entradas y salidas, los controles de temperatura, el uso supervisado de Elementos de Protección Individual (EPI's) y la vigilancia epidemiológica sobre el personal, entre otros. En la presente investigación, se plantea y valida el instrumento ERBioCov, que tiene la finalidad de valorar la proporción en la implementación de protocolos nuevos y tradicionales de higiene y bioseguridad, la cual fue aplicada en industrias de Lima, Perú en dos períodos de 2020 y 2021 . De acuerdo a los valores obtenidos, el 80,5\% de medidas de higiene y bioseguridad son aplicadas en la actualidad por las industrias estudiadas en Perú, en contraste con el $68,9 \%$ obtenido en los meses iniciales de la pandemia en 2020 , diferencia para la cual se comprobó una alta significancia estadística ( $\mathrm{p}=3,93 \mathrm{E}-06$; $\mathrm{t}=-5,887)$. Entre ellas, las medidas tradicionales tuvieron un mayor incremento en su aplicación entre ambos períodos estudiados, llegando de $65,6 \%$ al $80,8 \%(\mathrm{p}=0 ; \mathrm{t}=-6,544)$ en comparación de las medidas adicionales, que se mantuvieron en el rango de 78,4\% y $79,6 \%$ en 2020 y 2021, respectivamente ( $\mathrm{p}=0,7442 ; \mathrm{t}=-0,326)$. Igualmente, se obtuvo una alta tasa de prevención ante riesgos biológicos, cuantificada en 28 medidas aplicadas en un rango del $93 \%$ al $100 \%$ de las industrias participantes.

Palabras clave: COVID-19, Higiene, Bioseguridad, ERBioCov, Industria

\section{ABSTRACT}

The high risk of contagion and affectation by COVID-19 in industrial workers made it necessary to rethink the hygiene and biosafety protocols already established, with measures such as distancing from jobs, staggering entry and exit times, controls temperature, the supervised use of Individual Protection Elements (PPE's) and epidemiological surveillance on personnel, among others. In this research, the ERBioCov instrument is proposed and validated, which aims to assess the proportion in the implementation of new and traditional hygiene and biosafety protocols, which was applied in industries in Lima, Peru in two periods of 2020 and 2021. According to the values obtained, 80.5\% of hygiene and biosafety measures are currently applied by the industries studied in Peru, in contrast to the $68.9 \%$ obtained in the initial months of the pandemic in 2020 , difference for which a high statistical significance was verified ( $p=3,93 E-06 ; t-5.887)$. Among them, the traditional measures had a greater increase in their application between both periods studied, reaching from $65.6 \%$ to $80.8 \%$ ( $p=0 ; t=-6.544)$ compared to the additional measures, which remained at the range of $78.4 \%$ and $79.6 \%$ in 2020 and 2021, respectively ( $p=0.7442 ; t=-0.326)$. Likewise, a high rate of prevention against biological risks was obtained, quantified in 28 measures applied in a range of $93 \%$ to $100 \%$ of the participating industries.

Key words: COVID-19, Hygiene, Biosafety, ERBioCov, Industry

1 Universidad Privada del Norte (UPN). Lima, Perú 2 Universidad de San Martín de Porres (USMP). Lima, Perú 3 Universidad Inca Garcilaso de la Vega (UIGV). Lima, Perú

4 Universidad Nacional del Callao (UNAC). Callao, Perú

*Autor de Correspondencia: rdavila430@gmail.com

\section{Introducción}

Uno de los componentes más importantes de la industria, y en general de cualquier organización, es el elemento humano (Campero, 1990; Sucunza, 2004). Por ello, es vital que los trabajadores sean protegidos ante amenazas que 
vulneren su integridad, en especial si éstas pueden prevenirse, teniendo en cuenta una triada que comprende los aspectos social, productivo y económico. La primera consiste en valorar al trabajador de manera individual, apartándose del modelo que lo considera un recurso, y actuando en pro de sus derechos, siendo los más primordiales la vida y la salud (Gonzalez et al., 2007; Palomino, 2015). La segunda considera la productividad en función de la fuerza laboral, es decir, trabajadores saludables tendrán un mayor y mejor rendimiento, disminuyendo a su vez la necesidad de reposos o licencias (Davis et al., 2005; Loeppke et al., 2007). La tercera plantea que prevenir los riesgos en el entorno laboral es más rentable que la atención de las consecuencias en la salud de los trabajadores (Ruiz-Frutos et al., 2007), especialmente al tener en cuenta que en la región sólo el 15\% de ellos tienen acceso a servicios básicos de salud (OPS, 2010).

Este principio del cuidado del trabajador se relaciona estrechamente con el accionar de la salud ocupacional, la cual se define como "la promoción y mantenimiento del (...) bienestar físico, mental y social de los trabajadores en todas las ocupaciones mediante la prevención de las desviaciones de la salud, control de riesgos y la adaptación del trabajo a la gente, y la gente a sus puestos de trabajo" (OPS, 2010). Dentro de ella, se enmarcan los conceptos de higiene y bioseguridad, una práctica dual considerada la principal estrategia no médica para prevenir enfermedades profesionales, mediante la identificación, evaluación y control de los contaminantes de origen laboral (Concepción et al., 1996; Torres \& Carbonell, 2015), en especial de los agentes químicos y biológicos, que ponen en riesgo a los trabajadores de la industria (Niu, 2007).

Tal enfoque preventivo reviste mayor importancia al considerar los desafíos que representa la detección de riesgos biológicos dentro del entorno laboral, pues debido a su tamaño microscópico no pueden ser apreciados a simple vista, logrando en ocasiones afectar a uno o varios trabajadores antes de ser identificados. Según la Organización Panamericana de la Salud (OPS, 2015), dentro de estos agentes biológicos se encuentran bacterias, virus y parásitos patogénicos, además de ciertas toxinas de origen microbiano, que pueden tener efectos sobre la salud como infecciones e intoxicaciones, que pueden ser o no de origen zoonótico; las cuales pueden afectar clínicamente al tracto respiratorio, como en el caso del asma ocupacional, derivar en reacciones alérgicas, o desencadenar efectos sistémicos (EU-OSHA, 2017).

Algunas medidas de higiene y bioseguridad se han establecido con el fin disminuir los focos de riesgo, como el lavado y descontaminación de superficies, la prohibición del ingreso de animales domésticos, o el uso de rodenticidas. Sin embargo, se deben tener en cuenta dos aspectos adicionales, como lo son: las vías de transmisión y la exposición del trabajador; es decir, qué medios utiliza el patógeno o agente infeccioso para llegar al trabajador, y cómo penetran en el interior de su organismo. Aquellos riesgos de transmisión física que logran su entrada al organismo mediante ingestión o contacto dérmico, especialmente con piel lesionada o zonas mucosas expuestas, han hecho necesario el uso de prendas protectoras y protocolos de lavado corporal; por otra parte, ciertos agentes etiológicos ingresan de manera aerógena, para lo cual se usan las barreras faciales como mascarillas y filtros de ventilación; en otros casos la intervención vectorial es vital para la transmisión, motivando el uso de insectocutores (Concepción et al., 1996; Torres \& Carbonell, 2015; Aguilar, 2015).

De acuerdo a los datos obtenidos por Dávila Morán et al., (2021) en una investigación preliminar, la implementación de estas y otras medidas mejoraron la seguridad de los trabajadores de la industria, hasta la propagación global en 2020 del Sars-Cov-2, un nuevo coronavirus altamente patogénico, transmisible por contacto directo e indirecto con una persona infectada o por las gotas de secreciones humanas, y con capacidad de supervivencia en ropas, superficies y manos contaminadas. Al respecto, Leso et al. (2021) indican que la proximidad física derivada de la interacción laboral aumenta el riesgo de contraer la enfermedad, denominada COVID-19. Tales características, en conjunto, hicieron necesario replantear los protocolos de higiene y bioseguridad ya establecidos, con medidas como el distanciamiento en puestos de trabajo, el escalonamiento en horario de entradas y salidas, los controles de temperatura, el uso estricto y supervisado de Elementos de Protección Individual (EPI's), el reforzamiento en la vigilancia epidemiológica sobre el personal y las licencias preventivas a grupos trabajadores posterior al contacto con un infectado. En el caso de las tres últimas, se amplió su alcance en las industrias donde ya eran aplicadas.

\section{Metodología}

Se desarrolló un estudio descriptivo, de corte transversal y no probabilístico, entre junio de 2020 y abril de 2021; como parte de una gran investigación de los efectos de la COVID-19 en el sector industrial del Perú, descrita por Dávila Morán et al., (2021). En la fase inicial, mediante búsqueda en bases de datos digitales, se identificaron 38 industrias grandes y medianas de los sectores: alimentario, farmacéutico, producción animal o donde existe contacto con animales, localizadas en la provincia de Lima. Fue enviado un cuestionario a los correos electrónicos de estas organizaciones; donde se explicó el objetivo del estudio, indicando la intención de confidencialidad sobre los nombres de las industrias participantes, y solicitando respuesta a las siguientes preguntas: Primero, ¿En su organización se habían implementado 
medidas de higiene y bioseguridad antes de la llegada de la COVID-19? Segundo, ¿En su organización se tomaron o incrementaron medidas de higiene y bioseguridad posterior a la llegada de la COVID-19? Tercero, ¿Serían partícipes de una posterior encuesta más específica? Se obtuvo respuesta de 22 instituciones; de este grupo, la totalidad contestó afirmativamente a la primera pregunta, y el 86,4\% (n=19) manifestó haber implementado medidas adicionales de higiene y bioseguridad de manera consecuente a la pandemia por el nuevo coronavirus, dando además su consentimiento para continuar la investigación. Para la segunda fase se usó la escala "ERBio", propuesta y validada por Aguilar, (2015) la cual permite evaluar en 48 preguntas los riesgos biológicos a los que habitualmente pueden estar expuestas las personas dentro de las industrias. Al considerar la presencia de la COVID-19 en la nación peruana, con el subsecuente riesgo adicional que representa para el componente humano de la industria, se planteó un instrumento complementario desarrollado a partir del análisis cruzado de las recomendaciones de la Organización Mundial de la Salud (OMS, 2020a, b), la Organización Internacional del Trabajo (OIT, 2020), el Organismo Internacional de Normalización (ISO, 2020), la Administración de Seguridad y Salud Ocupacional estadounidense (OSHA, 2020) y el Ministerio de Salud de Perú (Minsa, 2020a,b) en concordancia a la metodología planteada por Dávila Morán et al., (2021). Este cuestionario consta de 17 ítems, puesto previamente a consideración de un grupo de 5 expertos con el fin de evaluar su válidez aparente (de contenido), mediante la retroalimentación controlada según los juicios individuales, sobre la base de la información disponible. A la opinión individual se asignaron puntajes por ítems usando la escala de Likert valorizada de 1 a 5, y se determinó la distancia de los puntos múltiples (DPP) mediante la siguiente ecuación

$$
\mathrm{DPP}=\sqrt{ }\left(\left(\mathrm{x}_{1}-\mathrm{y}_{1}\right)^{2}+\left(\mathrm{x}_{2}-\mathrm{y}_{2}\right)^{2}+\ldots+\left(\mathrm{x}_{\mathrm{n}}-\mathrm{y}_{\mathrm{n}}\right)^{2}\right)
$$

Una vez realizada la validación, considerando también el alfa de Cronbach, se procedió a sintetizar ambos instrumentos, generando así el instrumento ERBioCov con un total de 65 ítems y opciones de respuesta Afirmativa, Negativa o No aplicable, la cual fue enviada en agosto de 2020 a las 19 industrias extraídas de la fase inicial, haciendo uso de la plataforma Google Forms para la colección de los datos. 15 de ellas completaron el instrumento dentro de los 30 días posteriores, configurando así la muestra de nuestro estudio. La tabulación y análisis de la data obtenida se realizó entre octubre y diciembre de 2020. La última fase consistió en la visita presencial a las 15 organizaciones, llevada a cabo entre febrero y marzo de 2021, cumpliendo los protocolos de bioseguridad y garantizando el uso de los adecuados elementos de protección personal a los 3 entrevistadores, con el fin de corroborar y actualizar los resultados obtenidos en la fase anterior. Finalmente, los hallazgos fueron comparados y analizados con medidas estadísticas de tendencia central, chi cuadrado y t-student.

\section{Resultados}

La valoración de contenido de la escala medidas adicionales Cov mostró una distancia de los puntos múltiples DPP de 2,2 lo que permite considerarla adecuada para ser aplicada con fines de investigación, y cuyos ítems muestran una importancia atribuible (Figura 1) dentro del rango del 80\% (ítem 54) al 100\% (ítem 62). Adicionalmente, se halló un alfa de Cronbach de 0,87 que indica Alta consistencia del instrumento.

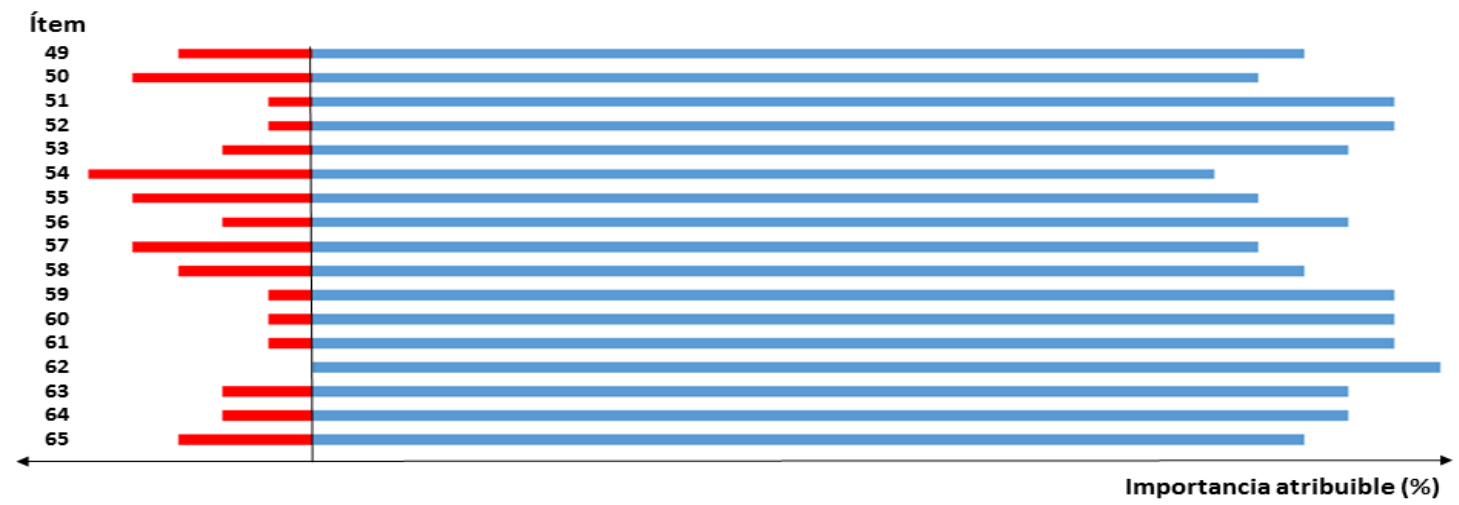

\section{Figura 1. Validez de contenido de la escala Medidas Adicionales Cov}

La tabla 1 muestra el total y la proporción de respuestas afirmativas sobre cada ítem, dadas por las industrias en 2020 y verificadas en 2021, sobre los métodos de higiene y bioseguridad implementados antes y durante la pandemia por COVID-19. De acuerdo a los valores obtenidos, el 80,5\% de medidas de higiene y bioseguridad son aplicadas en la actualidad por las industrias estudiadas en Perú, en contraste con el 68,9\% obtenido en los meses iniciales de la pandemia por COVID-19 en 2020, diferencia para la cual se comprobó una alta significancia estadística ( $\mathrm{p}=3$,93e-06; $\mathrm{t}=-5,887)$. Entre las medidas tradicionales que ampliaron su alcance o tuvieron mayor acogencia entre ambos períodos 
$(\mathrm{p}=0 ; \mathrm{t}=-6,544)$ están la implementación de un local o dispositivo para el almacenamiento sustancias desinfectantes $(\mathrm{p}=0,006 ; \mathrm{t}=-2,739)$, el protocolo de lavado y desinfección de manos $(\mathrm{p}=0,032 ; \mathrm{t}=-2,148)$, el control sobre la disponibilidad y el uso adecuado de EPI's $(\mathrm{p}=0,032 ; \mathrm{t}=-2,148)$, la adecuación de espacios exclusivos para la disposición de prendas de ropa contaminadas $(\mathrm{p}=0,028 ; \mathrm{t}=-2,477)$, el cumplimiento de un programa de gestión de residuos $(\mathrm{p}=0,032 ; \mathrm{t}=-2,148)$, y el conocimiento de los trabajadores sobre el grado de peligrosidad de los contaminantes biológicos pueden estar presentes en el lugar de trabajo $(\mathrm{p}=0,014 ; \mathrm{t}=-2,449)$. Por otra parte, dentro de las medidas adicionales implementadas ante la presencia del COVID-19 ( $\mathrm{p}=0.7442 ; \mathrm{t}=-0,326)$ la más destacada estadísticamente consistió en la correcta manipulación de los protectores faciales $(\mathrm{p}=0,068 ; \mathrm{t}=-1,826)$.

La vacunación contra la COVID-19, iniciada en territorio peruano en el primer trimestre de 2021 (Minsa, 2021), motivó los cambios estadísticamente más significativos en el resultado interanual del instrumento, consistentes en la disposición de un carnet de vacunación para cada trabajador ( $\mathrm{p}=0,002 ; \mathrm{t}=-3,098)$, el conocimiento de los trabajadores sobre los beneficios, ventajas e inconvenientes tanto de la vacunación como de la no vacunación $(\mathrm{p}=0,001 ; \mathrm{t}=-2,579)$ y la especificad sobre las fechas de próximas dosis en el carnet ( $\mathrm{p}=0,003 ; \mathrm{t}=-2,981)$. Por otra parte, en las industrias estudiadas, la medida adicional de escalonamiento en las horas de llegada y salida en los puntos de entrada y evacuación disminuyó de un $86,7 \%$ al $53,3 \%$ ( $\mathrm{p}=0,046 ; \mathrm{t}=1,992)$.

Tabla 1. Comparativo histórico de respuestas al instrumento ERBioCov

\begin{tabular}{|c|c|c|c|c|c|c|c|}
\hline \multirow[b]{2}{*}{ No. } & \multirow{2}{*}{$\begin{array}{l}\text { Ítem } \\
\text { Detalle }\end{array}$} & \multicolumn{2}{|c|}{$\begin{array}{l}\text { Respuestas } \\
\text { Afirmativas } \\
\text { en } 2020\end{array}$} & \multicolumn{2}{|c|}{$\begin{array}{l}\text { Respuestas } \\
\text { Afirmativas } \\
\text { en } 2021\end{array}$} & \multicolumn{2}{|c|}{ Pruebas estadísticas } \\
\hline & & $\mathrm{n}$ & $\%$ & $\mathrm{n}$ & $\%$ & Valor de $\mathrm{P}$ & Valor de $\mathrm{t}$ \\
\hline 1 & $\begin{array}{l}\text { ¿Existe un suelo de materiales impermeables, fácil de limpiar y de desinfectar, imputrescible y dispuesto de } \\
\text { forma tal que permita una salida fácil del agua; para evitar los olores? }\end{array}$ & 11 & 73,3 & 13 & 86,7 & 0,361 & $-0,913$ \\
\hline 2 & ¿Las paredes son lisas, resistentes e impermeables, recubiertas de un revestimiento lavable? & 12 & 80,0 & 13 & 86,7 & 0,624 & $-0,490$ \\
\hline 3 & $\begin{array}{l}\text { ¿Se dispone de dispositivos adecuados de protección contra los animales indeseables tales como insectos o } \\
\text { roedores (insectocutores y rodenticidas)? }\end{array}$ & 7 & 46,7 & 6 & 40,0 & 0,713 & 0,368 \\
\hline 4 & $\begin{array}{l}\text { ¿Se dispone de una instalación que permita el suministro de agua potable, a presión y en cantidad } \\
\text { suficiente? }\end{array}$ & 14 & 93,3 & 15 & 100,0 & 0,309 & $-1,017$ \\
\hline 5 & $\begin{array}{l}\text { ¿Se dispone de una instalación que proporcione una cantidad suficiente de agua potable caliente para ser } \\
\text { utilizada por los trabajadores? }\end{array}$ & 8 & 53,3 & 8 & 53,3 & 1 & 0 \\
\hline 6 & $\begin{array}{l}\text { ¿Se dispone de un número adecuado de vestuarios dotados de paredes y de suelos lisos, impermeables y } \\
\text { lavables, de lavabos, de duchas y de evacuatorios con agua corriente, equipados de manera que protejan } \\
\text { de una posible contaminación las partes limpias del edificio? }\end{array}$ & 10 & 66,7 & 10 & 66,7 & 1 & 0 \\
\hline 7 & $\begin{array}{l}\text { ¿Hay vestuarios provistos de dobles taquillas u otro sistema que impida que la ropa de trabajo se mezcle } \\
\text { con la ropa de calle? }\end{array}$ & 9 & 60,0 & 11 & 73,3 & 0,439 & $-0,775$ \\
\hline 8 & $\begin{array}{l}\text { En los cuartos vestuarios o de aseo chay duchas con servicio de agua caliente y fría? al menos } 1 \text { por cada } 10 \\
\text { trabajadores o fracción que trabajen en la misma jornada }\end{array}$ & 7 & 46,7 & 8 & 53,3 & 0,715 & $-0,365$ \\
\hline 9 & ¿Las duchas están aisladas en cabinas o compartimentos individuales? & 6 & 40,0 & 6 & 40,0 & 1 & 0 \\
\hline 10 & ¿Hay, al menos, un inodoro por cada 25 personas que trabajen en la misma jornada? & 12 & 80,0 & 12 & 80,0 & 1 & 0 \\
\hline 11 & ¿Los retretes son con descarga automática de agua corriente y están separados en cabinas aisladas? & 11 & 73,3 & 11 & 73,3 & 1 & 0 \\
\hline 12 & ¿Hay jabón desinfectante en lavabos y duchas? & 13 & 86,7 & 15 & 100,0 & 0,143 & $-1,464$ \\
\hline 13 & $\begin{array}{l}\text { ¿Los retretes están separados de los vestuarios o de las áreas de trabajo por un local o zona intermedia de } \\
\text { paso? }\end{array}$ & 6 & 40,0 & 6 & 40,0 & 1 & 0 \\
\hline 14 & $\begin{array}{l}\text { ¿Los retretes tienen acceso sencillo e inmediato (por ejemplo, no hay que ir a buscar una llave para poder } \\
\text { entrar en ellos)? }\end{array}$ & 14 & 93,3 & 14 & 93,3 & 1 & 0 \\
\hline 15 & ¿Los trabajadores tienen suficientes áreas de descanso? & 13 & 86,7 & 13 & 86,7 & 1 & 0 \\
\hline 16 & ¿Hay una sala de primeros auxilios? & 11 & 73,3 & 13 & 86,7 & 0,361 & $-0,913$ \\
\hline 17 & $\begin{array}{l}\text { ¿Se dispone de un local o dispositivo para el almacenamiento de detergentes, desinfectantes y sustancias } \\
\text { similares? }\end{array}$ & 9 & 60,0 & 15 & 100,0 & 0,006 & $-2,739$ \\
\hline 18 & $\begin{array}{l}\text { ¿Las instalaciones de oficinas, laboratorios de calidad, etc. se encuentran separados físicamente de las } \\
\text { instalaciones donde existe el riesgo biológico? }\end{array}$ & 11 & 73,3 & 11 & 73,3 & 1 & 0 \\
\hline 19 & $\begin{array}{l}\text { Si se dispone de naves (edificaciones) de ambiente controlado ¿estas cuentan con filtros para bacterias y } \\
\text { virus a la entrada de la toma de aire? }\end{array}$ & 5 & 33,3 & 9 & 60,0 & 0,143 & $-1,464$ \\
\hline 20 & ¿Los equipos de trabajo se limpian y/o desinfectan periódicamente? & 8 & 53,3 & 15 & 100,0 & 0,003 & $-3,022$ \\
\hline 21 & $\begin{array}{l}\text { ¿Todo material que se introduce en la instalación es lavado y desinfectado previamente, disponiendo de un } \\
\text { local específico para ello? }\end{array}$ & 10 & 66,7 & 14 & 93,3 & 0,068 & $-1,826$ \\
\hline 22 & ¿Se realiza una limpieza y desinfección general del lugar de trabajo de manera periódica? & 13 & 86,7 & 15 & 100,0 & 0.1432 & $-1,464$ \\
\hline 23 & ¿Existen sistemas de limpieza tipo lavabotas, lavamanos en zonas de entrada y salida a los locales? & 12 & 80,0 & 15 & 100,0 & 0,068 & $-1,826$ \\
\hline 24 & $\begin{array}{l}\text { ¿El personal se lava innatamente y cuidadosamente las manos y los brazos con agua y luego los } \\
\text { desinfectan? }\end{array}$ & 11 & 73,3 & 15 & 100,0 & 0,032 & $-2,148$ \\
\hline 25 & $\begin{array}{l}\text { ¿Los trabajadores, dentro de la jornada laboral, tienen } 10 \text { minutos para su aseo personal antes de la comida } \\
\text { y otros } 10 \text { minutos antes de abandonar el trabajo? }\end{array}$ & 9 & 60,0 & 12 & 80,0 & 0,232 & $-1,195$ \\
\hline 26 & $\begin{array}{l}\text { En caso de que haya exposiciones accidentales a materiales de riesgo, ¿Se lava la zona con agua y jabón } \\
\text { abundante y se declara inmediatamente el accidente o incidente? }\end{array}$ & 15 & 100,0 & 15 & 100,0 & 1 & 0 \\
\hline 27 & ¿Se evita la presencia de animales domésticos en el interior de la empresa? & 12 & 80,0 & 12 & 80,0 & 1 & 0 \\
\hline 28 & $\begin{array}{l}\text { ¿El empresario proporciona los equipos de protección y controla que son los adecuados y que se utilizan de } \\
\text { forma adecuada? }\end{array}$ & 11 & 73,3 & 15 & 100,0 & 0,032 & $-2,148$ \\
\hline 29 & ¿Los trabajadores conocen las características de las prendas y equipos de protección? & 12 & 80,0 & 15 & 100,0 & 0,068 & $-1,826$ \\
\hline 30 & ¿Hay un almacén o recipientes adecuados para guardar por separado la ropa sucia de la ropa limpia? & 5 & 33,3 & 11 & 73,3 & 0,028 & $-2,477$ \\
\hline 31 & $\begin{array}{l}\text { ¿Los equipos de protección se comprueban antes, o en todo caso después de cada uso, reparando y } \\
\text { sustituyendo los equipos defectuosos antes de su nuevo uso? }\end{array}$ & 12 & 80,0 & 15 & 100,0 & 0,068 & $-1,826$ \\
\hline
\end{tabular}




\begin{tabular}{|c|c|c|c|c|c|c|c|}
\hline \multirow[b]{2}{*}{ No. } & \multirow{2}{*}{ Ítem } & \multicolumn{2}{|c|}{$\begin{array}{l}\text { Respuestas } \\
\text { Afirmativas } \\
\text { en } 2020\end{array}$} & \multicolumn{2}{|c|}{$\begin{array}{l}\text { Respuestas } \\
\text { Afirmativas } \\
\text { en } 2021\end{array}$} & \multicolumn{2}{|c|}{ Pruebas estadísticas } \\
\hline & & $\mathrm{n}$ & $\%$ & $\mathrm{n}$ & $\%$ & Valor de $\mathrm{P}$ & Valor de $\mathrm{t}$ \\
\hline 32 & $\begin{array}{l}\text { Al salir de la zona de trabajo, ¿el trabajador se quita las ropas de trabajo y los equipos de protección } \\
\text { individual potencialmente contaminados y sucios y los guarda en un lugar que no contiene otras prendas? }\end{array}$ & 11 & 73,3 & 14 & 93,3 & 0,142 & $-1,470$ \\
\hline 33 & $\begin{array}{l}\text { ¿El empresario se responsabiliza del lavado y descontaminación / o destrucción de la ropa de trabajo y de } \\
\text { los EPI's? }\end{array}$ & 4 & 26,7 & 5 & 33,3 & 0,690 & $-0,398$ \\
\hline 34 & $\begin{array}{l}\text { ¿El empresario prohíbe, de manera rigurosa, que los trabajadores se lleven la ropa de trabajo y los EPI's a } \\
\text { su domicilio? }\end{array}$ & 3 & 20,0 & 6 & 40,0 & 0,232 & $-1,195$ \\
\hline 35 & $\begin{array}{l}\text { ¿Los trabajadores disponen de un carnet de vacunación que certifica que han recibido una dosis } \\
\text { determinada de las vacunas? }\end{array}$ & 6 & 40,0 & 14 & 93,3 & 0,002 & $-3,098$ \\
\hline 36 & $\begin{array}{l}\text { ¿Los trabajadores son informados sobre los beneficios, ventajas e inconvenientes tanto de la vacunación } \\
\text { como de la no vacunación? }\end{array}$ & 5 & 33,3 & 12 & 80,0 & 0,001 & $-2,579$ \\
\hline 37 & Si se dispone de los carnets de vacunación ¿aparecen las fechas de próximas vacunaciones? & 2 & 13,3 & 10 & 66,7 & 0,003 & $-2,981$ \\
\hline 38 & $\begin{array}{l}\text { ¿El trabajador conoce el grado de peligrosidad de los contaminantes biológicos que "están" o "pueden } \\
\text { estar" presentes en el lugar de trabajo? }\end{array}$ & 10 & 66,7 & 15 & 100,0 & 0,014 & $-2,449$ \\
\hline 39 & $\begin{array}{l}\text { ¿El empresario informa a todos los trabajadores expuestos de sus responsabilidades, para permitirles } \\
\text { desarrollar sus tareas y prevenir riesgos? }\end{array}$ & 15 & 100,0 & 15 & 100,0 & 1 & 0 \\
\hline 40 & $\begin{array}{l}\text { ¿Se planifican acciones formativas a todos los niveles para prevenir los riesgos a los que están expuestos los } \\
\text { trabajadores? }\end{array}$ & 10 & 66,7 & 14 & 93,3 & 0,068 & $-1,826$ \\
\hline 41 & $\begin{array}{l}\text { ¿La señal de peligro biológico está colocada en aquellos lugares o recipientes donde al entrar o el material } \\
\text { depositado puede dar lugar a una enfermedad infecciosa? }\end{array}$ & 12 & 80,0 & 12 & 80,0 & 1 & 0 \\
\hline 42 & $\begin{array}{l}\text { ¿Los trabajadores se lavan las manos y la piel expuesta antes de comer, beber, fumar, usar el teléfono o ir } \\
\text { al lavabo? }\end{array}$ & 11 & 73,3 & 14 & 93,3 & 0,142 & $-1,826$ \\
\hline 43 & ¿Está prohibido almacenar alimentos u otros productos de consumo humano en los locales de trabajo? & 12 & 80,0 & 12 & 80,0 & 1 & 0 \\
\hline 44 & ¿Hay un protocolo definido de primeros auxilios y se dispone de medios para llevarlo a cabo? & 9 & 60,0 & 10 & 66,7 & 0,705 & $-0,379$ \\
\hline 45 & $\begin{array}{l}\text { ¿Cuándo tienen alguna herida, los trabajadores, se la cubren con material impermeable, antes de empezar } \\
\text { la jornada laboral? }\end{array}$ & 10 & 66,7 & 10 & 66,7 & 1 & 0 \\
\hline 46 & ¿Hay y se cumple un programa de gestión de todos los residuos generados en el lugar de trabajo? & 11 & 73,3 & 15 & 100,0 & 0,032 & $-2,148$ \\
\hline 47 & ¿La higiene de locales se asegura mediante un procedimiento establecido de limpieza? & 13 & 86,7 & 15 & 100,0 & 0,143 & $-1,464$ \\
\hline 48 & $\begin{array}{l}\text { ¿Se usan mangueras de alta presión para limpiar las áreas que puedan estar contaminadas con materiales } \\
\text { de riesgo y considerar los líquidos de esta limpieza como materiales de riesgo? }\end{array}$ & 9 & 60,0 & 11 & 73,3 & 0,439 & $-0,775$ \\
\hline 49 & $\begin{array}{l}\text { ¿Proporciona y obliga el uso de desinfectante de manos mínimo de 60\% de etanol o } 70 \% \text { de alcohol } \\
\text { isopropílico? }\end{array}$ & 14 & 93,3 & 12 & 80,0 & 0,283 & 1,074 \\
\hline 50 & $\begin{array}{l}\text { ¿lmplementa técnicas eliminación de desechos eficaz, adecuada y frecuente, incluida la eliminación de } \\
\text { desechos separada y segura de EPI's? }\end{array}$ & 13 & 86,7 & 14 & 93,3 & 0,543 & $-0,609$ \\
\hline 51 & ¿Proporciona equipo de protección personal adecuado y / o mascarillas sin costo para el trabajador? & 15 & 100,0 & 15 & 100,0 & 1 & 0 \\
\hline 52 & ¿Insta a los trabajadores la correcta colocación de EPI y máscaras y su uso continuo? & 15 & 100,0 & 15 & 100,0 & 1 & 0 \\
\hline 53 & $\begin{array}{l}\text { ¿Insta a los trabajadores a evitar tocarse la cara o la máscara / cubierta de la cara, para evitar la } \\
\text { contaminación? }\end{array}$ & 12 & 80,0 & 15 & 100,0 & 0,068 & $-1,826$ \\
\hline 54 & ¿Escalona las horas de llegada y salida para reducir el hacinamiento en los puntos de entrada y evacuación? & 13 & 86,7 & 8 & 53,3 & 0,046 & 1,992 \\
\hline 55 & ¿Limita el número de pasajeros en los vehículos utilizados por la organización? & 8 & 53,3 & 6 & 40,0 & 0,464 & 0,732 \\
\hline 56 & $\begin{array}{l}\text { ¿Desinfecta con regularidad los dispositivos de seguridad táctiles, como teclados, lectores biométricos y } \\
\text { puntos de acceso electrónicos? }\end{array}$ & 13 & 86,7 & 12 & 80,0 & 0,624 & 0,490 \\
\hline 57 & ¿Reestructuró las estaciones de trabajo para permitir el distanciamiento físico entre cada estación? & 9 & 60,0 & 12 & 80,0 & 0,232 & $-1,195$ \\
\hline 58 & $\begin{array}{l}\text { ¿Realizó el bloqueo el uso de algunas estaciones de trabajo, o se incluyeron pantallas para separar a los } \\
\text { trabajadores si las estaciones de trabajo se fijan a una distancia menor a la recomendada? }\end{array}$ & 7 & 46,7 & 7 & 46,7 & 1 & 0 \\
\hline 59 & ¿Usa marcadores de piso o pared para indicar las pautas de distancia física recomendadas? & 6 & 40,0 & 7 & 46,7 & 0,713 & $-0,368$ \\
\hline 60 & $\begin{array}{l}\text { ¿Establece arreglos para evitar que personas potencialmente infecciosas ingresen al lugar de trabajo (por } \\
\text { ejemplo, proporcionando información antes de la visita o carteles que indiquen que las personas no deben } \\
\text { ingresar al lugar de trabajo con síntomas de COVID-19)? }\end{array}$ & 14 & 93,3 & 14 & 93,3 & 1 & 0 \\
\hline 61 & ¿En caso de recibir visitantes, son registrados para permitir el rastreo de contactos? & 13 & 86,7 & 12 & 80,0 & 0,624 & 0,490 \\
\hline 62 & ¿Existe una estación de desinfección de uso obligatorio anterior al ingreso de los visitantes? & 15 & 100,0 & 15 & 100,0 & 1 & 0 \\
\hline 63 & $\begin{array}{l}\text { ¿Exige a los visitantes que cumplan con las pautas de distanciamiento físico y otras medidas y controles de } \\
\text { seguridad como medidas frente al contacto con el público en los casos que sea necesario, en la } \\
\text { organización? }\end{array}$ & 14 & 93,3 & 14 & 93,3 & 1 & 0 \\
\hline 64 & $\begin{array}{l}\text { ¿Se usan carteles o letreros para informar al público sobre las medidas y controles de seguridad y cómo } \\
\text { mantener la distancia física? }\end{array}$ & 10 & 66,7 & 13 & 86,7 & 0,195 & $-1,295$ \\
\hline \multirow[t]{4}{*}{65} & ¿Se limita el número de miembros del público dentro de las instalaciones? & 9 & 60,0 & 12 & 80,0 & 0,232 & $-1,195$ \\
\hline & Subtotal escala ERBio (Medidas tradicionales ítems 1 a 48) & 472 & 65,6 & 582 & 80,8 & 0 & $-6,544$ \\
\hline & Subtotal escala Cov (Medidas adicionales ítems 49 al 65) & 200 & 78,4 & 203 & 79,6 & 0,7442 & $-0,326$ \\
\hline & Total escala ERBioCov (Todo el instrumento) & 672 & 68,92 & 785 & 80,51 & 3,93E-06 & $-5,887$ \\
\hline
\end{tabular}

\section{Discusión}

El aumento porcentual en la aplicación de medidas de higiene y bioseguridad a consecuencia de la pandemia COVID-19 en la industria peruana se explica en parte por la prevalencia de la enfermedad hasta la actualidad, con picos de contagio que refuerzan la percepción del riesgo, de acuerdo a Panovska-Griffiths et al., 2020 \& Heydari et al., 2021 aunado al interés económico de reestablecer o repuntar las actividades productivas, pero de una manera segura, al tener en cuenta que este virus generalmente ingresa al entorno laboral usando al trabajador como portador, ya sea como 
infectado o en sus elementos personales, convirtiéndolo en riesgo de contagio para sus compañeros (Baker et al., 2020; OMS, 2020a,b).

A diferencia de las conclusiones obtenidas por Aguilar-Elena et al., (2015) en tiempos de pre-pandemia, en la actual investigación se evidencia una alta tasa de prevención ante riesgos biológicos, ejemplificada por 28 medidas aplicadas en un rango del $93 \%$ al 100\% de las industrias participantes, que pueden ser agrupadas en las categorías de higiene y bioseguridad. Entre las primeras se encuentran: el suministro de agua potable; la disponibilidad de jabón desinfectante en lavabos y duchas; la disposición exclusiva para el almacenamiento de sustancias desinfectantes; la impieza y desinfección periódica de la locación y de los equipos de trabajo; la disposición de lavabotas y lavamanos en zonas de entrada y salida a los locales; el protocolo del lavado y desinfección de manos y brazos, con énfasis en los momentos previos a comer, beber, fumar o usar el teléfono; la desinfección de materiales introducidos al área de trabajo; el fácil acceso a instalaciones sanitarias; la ejecución de un programa de gestión de residuos y procedimientos establecidos de limpieza, que incluyen un protocolo para la disposición final de EPI's. La última categoría, de medidas de bioseguridad, incluyen la dotación de equipos de protección probados y adecuados, junto a la verificación de su uso continuo; el conocimiento de los trabajadores sobre la peligrosidad de los contaminantes biológicos que pueden estar presentes en el lugar de trabajo, y el correcto uso de EPI's; la disposición de un carnet de vacunación para cada trabajador; la planificación de acciones formativas para prevenir los riesgos a los que están expuestos los trabajadores; el uso de una estación de desinfección de uso obligatorio anterior al ingreso de los visitantes, instándolos a cumplir con las pautas de distanciamiento físico y otras medidas y controles de seguridad, y evitando el ingreso de personas potencialmente infectadas.

Sin embargo, en los resultados obtenidos también se aprecia la insuficiente aplicación de 3 medidas contra la COVID-19 recomendadas por la OMS, (2020a, b) la OIT, (2020) la ISO, (2020) la OSHA, (2020) y el Minsa, (2020a,b) que consisten en limitar el número de pasajeros en los vehículos utilizados por la organización, el bloqueo de algunas estaciones de trabajo o la inclusión pantallas para separar a los trabajadores, y el uso de marcadores de piso o pared para indicar las pautas de distancia física recomendadas; prácticas que disminuyeron en la comparación internanual, a consecuencia de las dificultades logísticas que conllevan.

Los beneficios de implementar medidas de bioseguridad en industrias peruanas durante la pandemia fueron demostrados por Pachas, (2021) quien logró relacionar la aplicación de los protocolos con la cantidad de contagios con COVID-19 en la empresa ( $\mathrm{p}=0.000292$ ), considerando la disminución en la proporción de casos positivos de $98.40 \%$ a $26.90 \%$ en ausencia y presencia de protocolos, respectivamente. Este autor también halló relaciones estadísticamente significativas con el bienestar laboral $(p=0.0015 \mathrm{E}-46)$ y el bienestar emocional ( $p$-valor= 4,0991E-62) de los trabajadores, lo cual es similiar a los precedentes establecidos por Peña \& Santos, (2018) quienes relacionaban el bienestar de los trabajadores con un lugar de trabajo seguro y saludable, logrado gracias a la aplicación de sistemas de gestión de seguridad y salud laborales.

De manera adicional, Leso et al. (2021) indican que la práctica de la higiene y bioseguridad en la industria se debe complementar con la identificación temprana de sujetos vulnerables, con el fin de orientar los procedimientos de evaluación y gestión de riesgos, las políticas preventivas colectivas, las acciones estrictas para grupos específicos de trabajadores, las decisiones sobre la colocación ocupacional de los empleados y las actividades de promoción de la salud. En coherencia a este planteamiento, Baker et al. (2020) agregan que la gran cantidad de personas empleadas en ocupaciones con exposición frecuente a infecciones y enfermedades, junto a la interacción laboral, resaltan la importancia de que todos los lugares de trabajo, además de la industria, desarrollen planes de respuesta al riesgo de COVID-19. Como beneficio adicional, será posible contrarrestar el nexo de transmisión de enfermedades a la comunidad.

Finalmente, el alto riesgo de contagio y afectación por COVID-19 en los trabajadores de la industria hizo necesario replantear los protocolos de higiene y bioseguridad ya establecidos. En la presente investigación, se planteó y validó el instrumento ERBioCov, con la finalidad de valorar la proporción en la implementación de protocolos nuevos y tradicionales de higiene y bioseguridad, la cual se aplicó en la investigación de 15 en industrias de Lima, Perú en dos períodos de 2020 y 2021. De acuerdo a los valores obtenidos, el 80,5\% de medidas de higiene y bioseguridad son aplicadas en la actualidad por las industrias estudiadas en Perú, en contraste con el 68,9\% obtenido en los meses iniciales de la pandemia en 2020, diferencia para la cual se comprobó una alta significancia estadística $(p=<0,001 ;$ $\mathrm{t}=-5,887)$. Entre ellas, las medidas tradicionales tuvieron un mayor incremento en su aplicación entre ambos períodos estudiados, llegando de $65,6 \%$ al $80,8 \%(\mathrm{p}=0 ; \mathrm{t}=-6,544)$ en comparación de las medidas adicionales, que se mantuvieron en el rango de 78,4\% y $79,6 \%$ en 2020 y 2021 respectivamente $(p=0,7442 ; \mathrm{t}=-0,326)$. Igualmente, se obtuvo una alta tasa de prevención ante riesgos biológicos, cuantificada en 28 medidas aplicadas en un rango del $93 \%$ al $100 \%$ de las industrias participantes. De acuerdo al análisis bibliográfico, estos hallazgos permiten considerar el beneficio para la salud de los trabajadores de esas industrias, y en consecuencia un menor riesgo de contagio hacia la comunidad. Las medidas transitorias, además, pueden servir de modelo para afrontar nuevos desafíos sanitarios dentro de la industria en el devenir del tiempo, tanto a nivel local, nacional, regional o global. 


\section{Conflictos de intereses}

Los autores declararon no tener conflictos de intereses.

\section{Agradecimientos}

A las instituciones participantes.

\section{Referencias}

Aguilar, R. (2015). Riesgos biológicos laborales: "ERBio", un nuevo método de evaluación teórica. Tesis para optar al título de Doctor en prevención de riesgos laborales. (Universidad Pública de Navarra). Disponible en: https://core.ac.uk/download/pdf/78049908.pdf

Aguilar-Elena, R., Campo-Barrio, A., Morchón, R., \& Martínez-Merino, V. (2015). Diferencias de protección frente al riesgo biológico laboral en función del tamaño de la empresa. Revista de Salud Pública, 17(2):195-207. https://doi.org/10.15446/rsap.v17n2.48288

Baker, M. G., Peckham, T. K., \& Seixas, N. S. (2020). Estimating the burden of United States workers exposed to infection or disease: A key factor in containing risk of COVID-19 infection. PloS one, 15(4):e0232452. https://doi.org/10.1371/journal.pone.0232452

Campero, M. (1990). El elemento humano en la administración de proyectos. Revista Ingeniería de Construcción, 9. Disponible en: http://www.ucla.edu.ve/dac/investigaci\%C3\%B3n/compendium8/proteccion.htm. (Acceso Marzo 2021).

Concepción, E., Viña, S., \& Montero, R. (1996). ¿ Dónde considerar a la seguridad e higiene ocupacional en los procesos de escalado de plantas farmacéuticas y biotecnológicas. Biotecnología Aplicada, 13(4). Disponible en: http://elfosscientiae.cigb.edu.cu/PDFs/Biotecnol\%20Apl/1996/13/4/p\%20307\%20-\%20310\%20.pdf (Acceso Julio 2020).

Dávila Morán, R. C. D., Pareja, C. G. G., Paredes, C. E. G., Nizama, J. L. R., \& Corzo, E. D. C. A. (2021). La pandemia de COVID-19: Amenaza biológica versus trabajo y sociedad. Boletín de Malariología y Salud Ambiental, 61(1), 29-37. https://doi.org/10.52808/bmsa.7e5.611.005

Davis, K., Collins, S., Doty, M., Ho, A. \& Holmgren, A. (2005). Salud y productividad entre los trabajadores $\begin{array}{llllll}\text { estadounidenses. Issue Brief (Commonw Fund), } 856 & \text { (856):1-10. Disponible en: }\end{array}$ https://www.commonwealthfund.org/sites/default/files/documents/_media_files_publications_issue brief_200 5 aug health and productivity among u $\mathrm{s}$ _ workers 856 davis hlt_productivity usworkers_pdf.pdf (Acceso Febrero 2021).

González, R., Hidalgo, G., \& Salazar, J. (2007). "Calidad de vida en el trabajo": un término de moda con problemas de conceptuación. Psicología y Salud, 17(1):115-123. https://doi.org/10.25009/pys.v17i1.746

ISO. (2020). ISO / PAS 45005. Gestión de la seguridad y la salud en el trabajo: directrices generales para un trabajo seguro durante la pandemia de COVID-19. https://www.iso.org/obp/ui/es/\#iso:std:iso:pas:45005:ed-1:v1:en (Acceso Julio 2020).

Leso, V., Fontana, L., \& Iavicoli, I. (2021). Susceptibility to Coronavirus (COVID-19) in Occupational Settings: The Complex Interplay between Individual and Workplace Factors. International journal of environmental research and public health, 18(3):1030. https://doi.org/10.3390/ijerph18031030

Loeppke, R., Taitel, M., Richling, D., Parry, T., Kessler, RC, Hymel, P. \& Konicki, D. (2007). Salud y productividad como estrategia empresarial. Revista de Medicina Ambiental y Ocupacional, 49(7):712-721. Disponible en: https://journals.lww.com/joem/Fulltext/2007/07000/The_Association_of_Medical_Conditions_and.4.aspx (Acceso Marzo 2021).

Minsa (Ministerio de Salud del Perú). (2020a). Coronavirus: medidas de prevención en el trabajo. Disponible en: https://www.gob.pe/8737-ministerio-de-salud-coronavirus-medidas-de-prevencion-en-el-trabajo (Acceso Julio 2021).

Minsa (Ministerio de Salud del Perú). (2020b). Resolución Ministerial N 448-2020-MINSA. Lineamientos para la Vigilancia, Prevención y Control de la salud de los trabajadores. Disponible en: https://www.gob.pe/institucion/minsa/normas-legales/744524-448-2020-minsa (Acceso Julio 2021). 
Minsa (Ministerio de Salud del Perú). (2021). Campaña Nacional de Vacunación contra la COVID-19. Disponible en: http://www.minsa.gob.pe/vacuna-covid-19/ (Acceso Marzo 2021).

Niu, S. (2007). Reconocimiento del origen laboral de las enfermedades causadas por agentes biológicos: una perspectiva de la OIT. Riesgos laborales por agentes biológicos: Afrontando los desafíos. Disponible en: https://osha.europa.eu/sites/default/files/seminars/documents/en/seminars/occupational-risks-from-biologicalagents/speech-venues/speeches/recognition-of-work-related-origin-of-diseases-caused-by-biological-agents2013-an-ilo-perspective/Presentation\%20by\%20Shengli\%20Niu.ppt (Acceso Marzo 2021).

OIT. (2020). Las normas de la OIT y la COVID-19 (coronavirus). Disponible en: https://www.ilo.org/wcmsp5/groups/public/---ed_norm/---normes/documents/publication/wcms_739939.pdf (Acceso Julio 2020).

OMS. (2020a). Especificaciones técnicas para el equipo de protección personal frente a la COVID-19. Disponible en: https://apps.who.int/iris/bitstream/handle/10665/339601/WHO-2019-nCoVPPE-specifications-2020.1-spa.pdf (Acceso Junio 2020).

OMS. (2020b). Getting your workplace ready for COVID-19. Disponible en: https://www.who.int/docs/defaultsource/coronaviruse/getting-workplace-ready-for-covid-19.pdf (Acceso Julio 2020).

OPS. (2010). Salud de los Trabajadores: Recursos. Disponible en: https://www3.paho.org/hq/index.php?option=com_content\&view=article\&id=1527: workers-healthresources\&Itemid=1349\&showall=1\&lang=es (Acceso Marzo 2021).

OPS. (2015). Category Index: HACCP. Clasificación de los peligros. Disponible en: https://www3.paho.org/hq/index.php?option=com_content\&view=article\&id=10837:2015-clasificacionpeligros\&Itemid=41432\&lang=en (Acceso Marzo 2021).

OSHA (Occupational Safety and Health Administration). (2020). Diez medidas que todos los lugares de trabajo pueden tomar para prevenir la exposición al Coronavirus. Disponible en: https://www.osha.gov/sites/default/files/publications/OSHA3995.pdf (Acceso Julio 2021).

Palomino, T. (2015). El trabajador: ¿Recurso humano? Gaceta Laboral, 21(3):335-343. Disponible en: http://www.redalyc.org/articulo.oa?id=33643814005 (Acceso Marzo 2021).

Ruiz-Frutos, C., García, A. M., Delclós, J., \& Benavides, F. G. (2007). Salud laboral: conceptos y técnicas para la prevención de riesgos laborales. Revista española de salud pública, 81(3):327-328. Disponible en: https://scielo.isciii.es/scielo.php?script=sci arttext\&pid=S1135-57272007000300009 (Acceso Marzo 2021).

Sucunza Saldise, F. (2004). La importancia de las personas. Qualitas hodie: Excelencia, desarrollo sostenible e innovación, $\quad 96: 80-81 . \quad$ ISSN $1133-2417 . \quad$ Disponible en: https://dialnet.unirioja.es/servlet/articulo?codigo=891323 (Acceso Marzo 2021).

Torres, A., \& Carbonell, A. (2015). Análisis comparativo entre los principios de bioseguridad y de seguridad en la industria. Revista Cubana de Salud y Trabajo, 16(3):3-11. Disponible en: https://www.medigraphic.com/cgibin/new/resumen.cgi?IDARTICULO=63272 (Acceso Marzo 2021).

EU-OSHA (Agencia Europea para la seguridad y la Salud en el Trabajo). (2017). Agentes biologicos. Disponible en: https://oshwiki.eu/wiki/Biological_agents (Acceso Febrero 2021).

Panovska-Griffiths, J., Kerr, C. C., Stuart, R. M., Mistry, D., Klein, D. J., Viner, R. M., \& Bonell, C. (2020). Determining the optimal strategy for reopening schools, the impact of test and trace interventions, and the risk of occurrence of a second COVID-19 epidemic wave in the UK: a modelling study. The Lancet. Child \& adolescent health, 4(11):817-827. https://doi.org/10.1016/S2352-4642(20)30250-9

Heydari, S. T., Zarei, L., Sadati, A. K., Moradi, N., Akbari, M., Mehralian, G., \& Lankarani, K. B. (2021). The effect of risk communication on preventive and protective Behaviours during the COVID-19 outbreak: mediating role of risk perception. BMC public health, 21(1):54. https://doi.org/10.1186/s12889-020-10125-5

Pachas, L. (2021). Implementación de un plan de seguridad y salud en el trabajo para prevenir los contagios de COVID19 en una industria de alimentos ubicada en el Callao, 2020. (Tesis de pregrado, Universidad Nacional Mayor de San Marcos, Facultad de Ingeniería Industrial, Escuela Profesional de Ingeniería Industrial). Repositorio institucional Cybertesis UNMSM. Disponible en: https://cybertesis.unmsm.edu.pe/handle/20.500.12672/16593 (Acceso Marzo 2021).

Peña, K., \& Santos, I. (2018). Diseño del Sistema de Gestión de Seguridad y Salud en el Trabajo en una empresa agroindustrial en Tambogrande, Piura, Perú. (Tesis de pregrado, Universidad de Piura, Facultad de Ingeniería. 


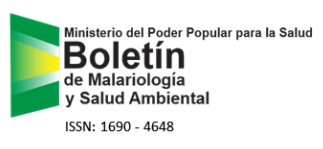

Programa

Académico de

Ingeniería

Industrial

Julio-Septiembre 2021, Vol. LXI (2): 78-86

https://pirhua.udep.edu.pe/handle/11042/3637 (Acceso Marzo 2021). 